\title{
Unipolar transport and shot noise in metal-semiconductor-metal structures
}

\author{
G. Gomila ${ }^{\text {a) }}$ \\ Centre de Recerca en Bioelectrònica i Nanobiociència and Departament d'Electrònica-Universitat \\ de Barcelona, Laboratori de Nanobioenginyeria-Parc Científic de Barcelona, C/ Josep Samitier 1-5, \\ E-08028 Barcelona, Spain \\ I. R. Cantalapiedra \\ Departament de Física Aplicada, Universitat Politècnica de Catalunya, Av. Dr. Marañon 44, \\ E-08028 Barcelona, Spain \\ L. Reggiani \\ INFM-National Nanotechnology Laboratory and Dipartimento di Ingegneria dell'Innovazione, \\ Università di Lecce, Via Arnesano s/n, I-73100, Lecce, Italy
}

(Received 24 July 2002; accepted 9 October 2002)

\begin{abstract}
We carry out a self-consistent analytical theory of unipolar current and noise properties of metalsemiconductor-metal structures made of highly resistive semiconductors in the presence of an applied bias of arbitrary strength. By including the effects of the diffusion current we succeed in studying the whole range of carrier injection conditions going from low level injection, where the structure behaves as a linear resistor, to high level injection, where the structure behaves as a space charge limited diode. We show that these structures display shot noise at the highest voltages. Remarkably the crossover from Nyquist noise to shot noise exhibits a complicated behavior with increasing current where an initial square root dependence (double thermal noise) is followed by a cubic power law. (C) 2003 American Institute of Physics. [DOI: 10.1063/1.1525863]
\end{abstract}

\section{INTRODUCTION}

Metal-semiconductor-metal structures have been of fundamental and applied interest since the birth of the physics of semiconductor devices. Here we concentrate on the relevant case of high resistivity semiconductors where metals usually form ohmic or injecting contacts. ${ }^{1}$ A common feature of these structures is that carrier transport is mainly limited by diffusion, with thermionic emission playing a negligible role. Furthermore, when a bias voltage is applied to these structures, they can behave either as a linear resistor (if carrier injection from the contacts is negligibly small) or as nonlinear space charge limited device (if carrier injection from the contacts is extremely high). ${ }^{2}$ Therefore, these structures still offer excellent opportunities to study current noise properties induced by diffusion noise in a variety of transport conditions.

The present knowledge of the noise properties of these structures can be summarized as follows. At thermodynamic equilibrium, the low frequency spectral density of current fluctuations, $S_{I}^{\mathrm{eq}}(0)$, is given by

$$
S_{I}^{\mathrm{eq}}(0)=\frac{4 k_{B} T}{R^{\mathrm{eq}}},
$$

in agreement with the Nyquist theorem. ${ }^{3}$ Here, $R^{\text {eq }}$ is the equilibrium device resistance, $T$ the bath temperature, and $k_{B}$ the Boltzmann constant.

\footnotetext{
${ }^{a)}$ Electronic mail: ggomila@pcb.ub.es
}

For departures from equilibrium, when an external bias (voltage or current) is applied, the Nyquist relation no longer holds and deviations from Eq. (1) are expected. These deviations have been studied in detail in two limiting cases, namely: (i) when the structure behaves as a space charge limited diode (with a strong inhomogeneous profile of the free carrier density), ${ }^{4-6}$ and (ii) when the structure behaves as a linear resistor (with a homogeneous profile of the free carrier density). ${ }^{7}$

In the former case (i) the current $I$ displays a quadratic dependence on the applied voltage $V$ (Mott-Gurney law) $I$ $=\beta V^{2}$, where $\beta$ is a sample dependent parameter. Accordingly, the low frequency spectral density of current fluctuations was found to take the form ${ }^{4-6}$

$$
S_{I}(0)=\frac{8 k_{B} T}{Z(0)}=16 k_{B} T \beta^{1 / 2} I^{1 / 2},
$$

where $Z(0)$ is the low frequency impedance (differential resistance). Considering the form of the first equality, this type of noise was called double thermal noise. ${ }^{8}$ The crossover between Nyquist noise and high voltage space charge limited conditions is summarized by the formula

$$
S_{I}(0)=4 k_{B} T \frac{V}{I}\left(\frac{d I}{d V}\right)^{2}
$$

Highly resistive structures under strong carrier injection conditions have been used to successfully test experimentally this prediction. ${ }^{8}$

In the latter case (ii) the structure behaves as a linear resistor and displays a resistance $R=L /(q A \mu \bar{n})$, where $A$ is 
the cross sectional area, $q$ the carrier charge, $\mu$ the mobility, and $\bar{n}$ the free carrier density (the bar denotes an average with respect to fluctuations). When the applied bias is high enough to make the transit time due to drift $\tau_{T}=L^{2} / \mu V$ significantly shorter than the dielectric relaxation time $\tau_{d}$ $=\epsilon / q \mu \bar{n}$, where $\epsilon$ is the dielectric permittivity, the structure was predicted to exhibit shot noise ${ }^{7}$ with

$$
S_{I}(0)=2 q \bar{I} \text {. }
$$

The above results show two completely different behaviors for the nonequilibrium current noise properties of metal-semiconductor-metal structures depending on whether the stationary free carrier distribution is strongly inhomogeneous (double thermal noise) or homogeneous (shot noise). The physical reason for such a discrepancy of behavior is an intriguing problem at present. In the authors opinion its origin can be traced back to the theoretical difficulty of accounting for the effects of the diffusion current on the nonequilibrium current fluctuations in the whole range of different carrier injection conditions.

The purpose of the present paper is to overcome such a difficulty by developing an analytical theory for the nonequilibrium current noise properties of metal-semiconductormetal structures made of highly resistive semiconductors that includes properly the effects of the diffusion current. The theory is valid in the whole range of physical conditions going from homogeneous to highly inhomogeneous free carrier distributions, thus allowing us to describe in a continuous manner the current spectral density as a function of the applied bias for different levels of current injection.

The article is organized as follows. Section II describes the system under study. Section III presents the physical model used to describe the low frequency transport and the nonequilibrium current fluctuation properties. Section IV is devoted to analyzing the charge transport properties of the device, while Sec. V analyzes its nonequilibrium current noise. In particular, we analyze the effects of space charge on the shot noise properties. Section VI draws the main conclusions of our investigation. Two appendixes provide the most technical derivations.

\section{SYSTEM UNDER STUDY}

The system under study consists of an active region made by a nondegenerate $n$-type semiconducting material sandwiched between two metal plates that act as contacts. The semiconductor is assumed to be lightly doped with a donor density $N_{D}$. All donors are assumed to be ionized at the considered temperatures (the case of a $p$-type semiconductor can be analogously considered). To justify a onedimensional electrostatic treatment in the $x$ direction and to neglect the effects of the boundaries in the $y$ and $z$ directions the transversal size of the sample is assumed to be much larger than the characteristic Debye screening length. The metals are assumed to form ohmic injecting contacts so that the voltage drop inside them, or equivalently, the contact resistance, can be neglected to a good approximation. Accordingly, when a voltage is applied to the structure, all the potential drop takes place inside the semiconductor between positions $x=0$ and $x=L$, and the contacts can be excluded from consideration. Depending on the parameter values of the contacts homogeneous as well as inhomogeneous conditions will be studied.

We assume that inelastic scattering processes with phonons are dominant, so that carrier thermalization at the bath temperature holds at any point. Accordingly, for the applied bias considered here no carrier heating takes place which allows us to use a field independent electron mobility and diffusivity.

\section{PHYSICAL MODEL}

\section{A. Charge transport}

The transport approach appropriate to describe the electrical properties of the metal-semiconductor-metal structure under study consists of the standard drift-diffusion current equation self-consistently coupled to the Poisson equation and supplemented by appropriate boundary conditions. The current drift-diffusion equation reads ${ }^{1}$

$$
\overline{\bar{I}}=q \bar{n}(x) \mu \bar{E}(x)+q D \frac{d \bar{n}(x)}{d x},
$$

where $A$ is the cross sectional area, $\bar{n}(x)$ the local carrier density, $\mu$ the mobility (assumed to be field independent), $\bar{E}(x)$ the local electric field, and $D$ the diffusion coefficient (related to the mobility through Einstein's relation $D / \mu$ $=k_{B} T / q$, where nondegenerate statistics are assumed). The bar denotes an average with respect to fluctuations. The Poisson equation is as usual

$$
\frac{d \bar{E}(x)}{d x}=\frac{q}{\epsilon}\left[N_{D}-\bar{n}(x)\right],
$$

with $\epsilon$ the static dielectric constant of the semiconducting material. The appropriate boundary conditions to describe the ohmic injecting contacts are given by (see Appendix A)

$$
\bar{n}(0)=\bar{n}(L)=n_{c},
$$

where the carrier density at the contacts $n_{c}$ is independent of the applied bias and given by (see Appendix A)

$$
n_{c}=N_{C} \exp \left(-\frac{q \phi_{b n}}{k_{B} T}\right) .
$$

Here, $N_{C}$ is the effective density of states in the conduction band and $\phi_{b n}$ the metal-semiconductor barrier height. For values of the contact parameters such as $n_{c}=N_{D}$ the stationary free carrier distribution is homogeneous, while for values such as $n_{c}>N_{D}$ there is net carrier injection from the contacts and the stationary carrier distribution is inhomogeneous.

Equations (5) and (6) can be combined into a single equation for the electric field

$$
-D \frac{d^{2} \bar{E}(x)}{d x^{2}}-\mu \bar{E}(x)\left[\frac{d \bar{E}(x)}{d x}-\frac{q N_{D}}{\epsilon}\right]=\frac{\bar{I}}{A}
$$

subject to the boundary conditions 


$$
\frac{d \bar{E}(0)}{d x}=\frac{d \bar{E}(L)}{d x}=\frac{q}{\epsilon}\left[N_{D}-n_{c}\right] .
$$

\section{B. Current fluctuations}

The only source of fluctuations in the system is related to the diffusion of carriers inside the structure. Accordingly, the low frequency noise properties (beyond $1 / f$ noise) can be described through a drift-diffusion-Langevin model. ${ }^{9-11}$ It consists of the linearized version of the transport model presented in the previous section supplemented by the appropriate Langevin source which describes the diffusion noise. The current equation in explicit form reads ${ }^{9-11}$

$$
\begin{aligned}
\frac{\delta I(t)}{A}= & q \mu \bar{E}(x) \delta n_{x}(t)+q \bar{n}(x) \mu \delta E_{x}(t) \\
& +q D \frac{d \delta n_{x}(t)}{d x}+\frac{\delta I_{x}(t)}{A},
\end{aligned}
$$

where $\delta E_{x}(t)$ and $\delta n_{x}(t)$ refer to the fluctuations of electric field and number density at point $x$, respectively, and $\delta I(t)$ refers to the fluctuations of the total current. In Eq. (11), consistently with the low-frequency limit taken here, the displacement current is neglected. Moreover, $\delta I_{x}(t)$ is a Langevin source associated with the fluctuations of the current induced by the diffusion of carriers inside the sample. It has zero mean and low frequency spectral density, ${ }^{9-11}$

$$
2 \int_{-\infty}^{+\infty} d t\left\langle\delta I_{x}(t) \delta I_{x^{\prime}}\left(t^{\prime}\right)\right\rangle=K(x) \delta\left(x-x^{\prime}\right),
$$

with brackets indicating ensemble average, and

$$
K(x)=4 q A k_{B} T \mu \bar{n}(x)=4 A q^{2} D \bar{n}(x),
$$

where in the last equality use is made of the Einstein relation. Finally, the linearized Poisson equation is given by

$$
\frac{d \delta E_{x}(t)}{d x}=-\frac{q}{\epsilon} \delta n_{x}(t),
$$

and the linearized boundary conditions by (see Appendix A)

$$
\delta n_{L}(t)=\delta n_{0}(t)=0 .
$$

In analogy with the case of the transport equations, we can combine Eqs. (11) and (14) into a single equation for the electric field fluctuation of the form

$$
\begin{aligned}
\frac{\delta I(t)-\delta I_{x}(t)}{A}= & -D \frac{d^{2} \delta E_{x}(t)}{d x^{2}}-\mu \bar{E}(x) \frac{d \delta E_{x}(t)}{d x} \\
& -\mu \delta E_{x}(t)\left[\frac{d \bar{E}(x)}{d x}-\frac{q N_{D}}{\epsilon}\right],
\end{aligned}
$$

with boundary conditions

$$
\left.\frac{d \delta E_{x}(t)}{d x}\right|_{0}=\left.\frac{d \delta E_{x}(t)}{d x}\right|_{L}=0 .
$$

\section{STATIONARY SPATIAL PROFILES AND CURRENT-VOLTAGE CHARACTERISTICS}

In this section we present the results concerning the transport properties for the structure under study calculated from the model presented in Sec. III A. In particular we will focus on the transition from homogeneous to inhomogeneous conditions. To this end, for given properties of the semiconductor we will vary the contact density from $n_{c}=N_{D}$ (corresponding to an homogeneous condition with ohmic contacts) to values $n_{c}>N_{D}$ (corresponding to inhomogeneous conditions with injecting contacts). Note that for given properties of the semiconductor, varying $n_{c}$ corresponds to varying the contact barrier height $\phi_{b n}$, i.e., changing the material for the metal contact.

In what follows, the results are conveniently discussed in terms of the values of two dimensionless parameters

$$
l=L / L_{D} \quad \text { and } \alpha=n_{c} / N_{D},
$$

with $L_{D}=\left(k_{B} T \epsilon / q^{2} N_{D}\right)^{1 / 2}$ being the Debye screening length associated with the semiconducting material. The parameter $l$ depends only on the properties of the given semiconductor, while $\alpha$ depends also on the contact properties, thus being independent variables. Since the nonequilibrium current noise properties of devices with homogeneous stationary free carrier density profiles were shown to change qualitatively when passing from $l<1$ to $l>1$, in what follows we will treat these two cases separately.

\section{A. Homogeneous solution}

For $\alpha=1\left(n_{c}=N_{D}\right)$, and any value of $l$, there exists a trivial homogeneous solution to the model presented in Sec. III A, namely

$$
\bar{n}(x)=N_{D}, \quad \bar{E}(x)=\frac{\bar{I}}{q A \mu N_{D}} .
$$

Accordingly, the current-voltage $(I-V)$ characteristics are linear and satisfies Ohm's law $\bar{I}=V / R_{\text {bulk }}$, with $R_{\text {bulk }}$ $=L /\left(q A \mu N_{D}\right)$ being the semiconductor resistance.

\section{B. Inhomogeneous solution}

For $\alpha>1\left(n_{c}>N_{D}\right)$ the solution to the model presented in Sec. III A is spatially inhomogeneous and cannot be obtained in a closed analytical form. Only under asymptotic conditions it is possible to derive approximate analytical expressions $^{2,6}$ (see also Appendix B). In any case, an exact solution of the transport model can be obtained by numerical integration following this procedure. One first solves numerically Eq. (9) subject to the boundary conditions in Eq. (10). Having found the electric field profile $\bar{E}(x)$, the carrier density profile $\bar{n}(x)$ is obtained from the Poisson equation (6). Furthermore, for a given value of the current $\bar{I}$, the applied voltage is found as $\bar{V}=\int_{0}^{L} d x \bar{E}(x)$, from where the $I-V$ characteristic is obtained.

In what follows we discuss the main features of the inhomogeneous solution. To this end we will consider two particular values of $l$, namely $l=0.1$ and $l=50$, as representative examples of the behavior observed for $l<1$ and $l>1$, 

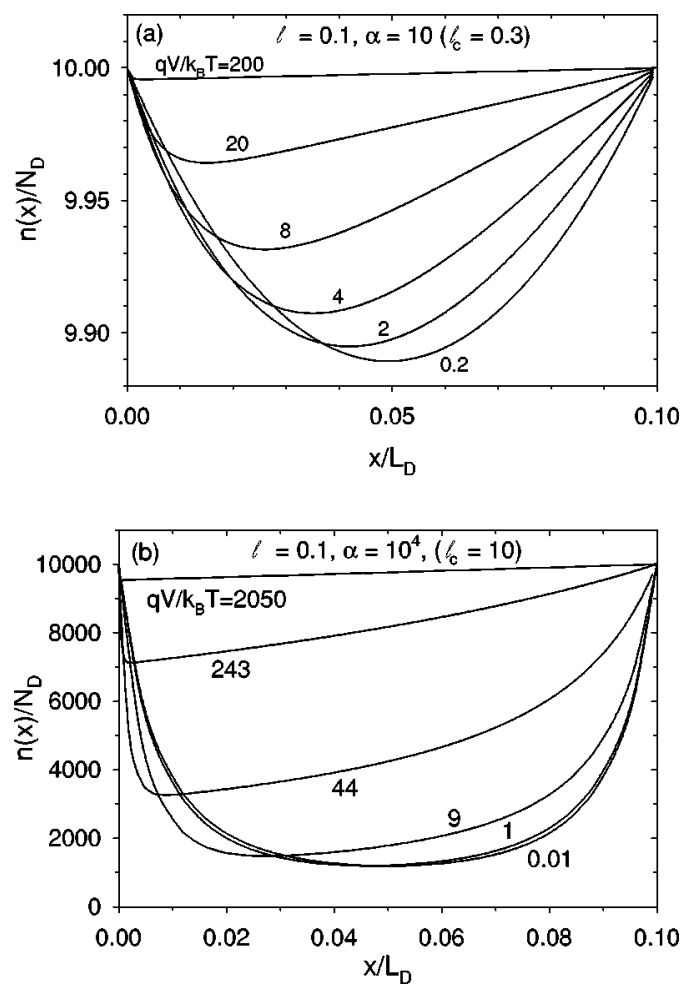

FIG. 1. Free carrier density profiles normalized to the donor density for several values of the applied bias, for $l=0.1$ and (a) $\alpha=10\left(l_{c}=0.3\right)$ and (b) $\alpha=10^{4}\left(l_{c}=10\right)$.

respectively. In both cases, the values of $\alpha$ will be varied in the range $1 \leqslant \alpha \leqslant 10^{4}$, thus allowing us to explore both slightly and highly inhomogeneous situations.

Figure 1 displays the stationary free-carrier density profiles for $l=0.1$ for several values of the applied bias and for $\alpha=10$ [Fig. 1(a)] and $\alpha=10^{4}$ [Fig. 1(b)]. As can be seen, the stationary free carrier density profiles are inhomogeneous and depend on the applied bias value.

For $\alpha=10$ [Fig. 1(a)] the degree of inhomogeneity is rather small being at most about $1 \%$, and the free carrier density departs more significantly from $n_{c}$ the lower the value of the applied voltage. The physical reason for this behavior can be found in the fact that the characteristic Debye screening length of the sample, roughly determined by $n_{c}$, i.e., $L_{D c}=\left(k_{B} T \epsilon / q^{2} n_{c}\right)^{1 / 2}$, is larger than the sample length $L\left(l_{c} \equiv L / L_{D c}=l \alpha^{1 / 2}=0.1 \times 10^{1 / 2} \sim 0.3\right)$ thus not allowing the free carrier density to relax from its contact value $n_{c}$ to its bulk value $N_{D}$, i.e., to the local charge neutral state. Similar behaviors appears for other values of $l<1$ and $\alpha$ $>1$ as long as $l_{c}=l \alpha^{1 / 2} \lesssim 1$.

For $\alpha=10^{4}$ [Fig. 1(b)] the degree of inhomogeneity becomes appreciable being up to about a factor of 10 . For low applied bias $\bar{n}(x)$ is almost symmetric and departs significantly from $n_{c}$. The reason is that the characteristic Debye screening length, which can be roughly approximated by $L_{D c}$, is shorter than the sample length $L\left(l_{c}=l \alpha^{1 / 2}=0.1\right.$ $\times 10^{2}=10$ ), thus allowing relaxation towards the charge neutral state. However, due to the small value of $l_{c}$, complete relaxation of $\bar{n}(x)$ to the value $N_{D}$ is not reached. At increasing applied bias, a net injection of carriers takes place

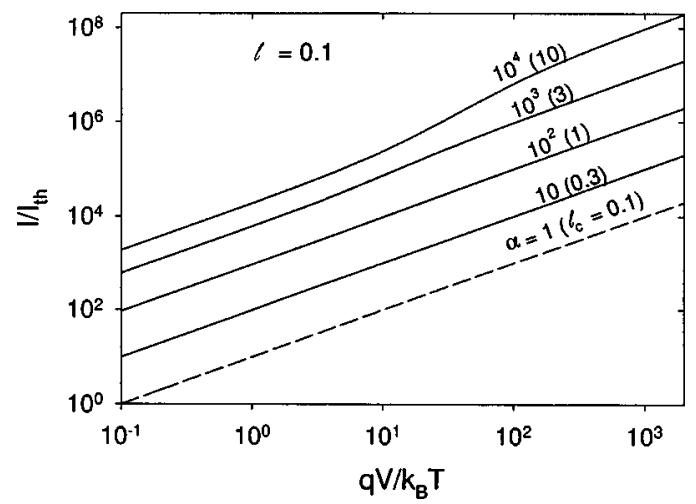

FIG. 2. Current-voltage characteristics for $l=0.1$ and several values of $\alpha$ (the corresponding value of $l_{c}$ is displayed for each curve). The dashed line corresponds to the homogeneous solution.

resulting in an increase of the values of $\bar{n}(x)$ and in a rather asymmetric profile. Finally, for the highest applied biases carrier injection no longer takes place, and the density distribution is almost homogeneous with a value close to the contact value $n_{c}$. Similar behaviors are observed for other values of $l<1$ and $\alpha>1$ as long as $l_{c}=l \alpha^{1 / 2}>1$.

The current-voltage characteristics for $l=0.1$ and for $\alpha$ ranging from 1 to $10^{4}$ are displayed in Fig. 2. For $\alpha \leqslant 10^{2}$, the $I-V$ characteristics are found to remain linear with a resistance given by $R_{c}=L /\left(q A \mu n_{c}\right)$. This linear behavior can be understood by noting that for these values of $\alpha$ the free carrier density profile is quasihomogeneous with a value approximately equal to $n_{c}$ and almost independent of the applied bias [see Fig. 1(a)]. Similar behaviors are found for other values of $l<1$ and $\alpha>1$ as long as $l_{c}=l \alpha^{1 / 2} \lesssim 1$. For $\alpha=10^{3}$ and $10^{4}$ the $I-V$ characteristics in Fig. 2 display a superlinear behavior in an intermediate range of applied voltages, while it is linear at the lowest and highest applied bias. The resistance at the lowest bias, $R_{\text {low }}$, satisfies $R_{c} \leqslant R_{\text {low }}$ $\leqslant R_{\text {bulk }}$, since in this range of bias the free carrier density profile is almost independent of bias and satisfies $n_{c} \leqslant \bar{n}(x)$ $\leqslant N_{D}$ [see Fig. 1(b)]. Furthermore, the resistance for high applied bias $R_{\text {high }}$ is almost equal to $R_{c}$, since in this range of bias the density profiles are almost independent of bias and given by $\bar{n}(x) \sim n_{c}$ [see Fig. 1(b)]. Finally, the superlinear behavior is due to the net injection of carriers taking place in this bias regime [see Fig. 1(b)] thus resulting in an increase of the conductivity of the sample. Similar behaviors appear for other values of $l<1$ and $\alpha>1$ as long as $l_{c}$ $=l \alpha^{1 / 2}>1$.

Let us now consider the case of $l=50$. Figure 3 reports the stationary free carrier density profile for $\alpha=10^{3}$ and several values of the applied bias. The qualitative behavior of the profiles is similar to those observed in Fig. 1(b), since both cases correspond to situations in which $l_{c}>1$ (in the present case $l_{c}=l \alpha^{1 / 2}=50 \times 10^{3 / 2} \sim 1581$ ). The main difference between these figures is that in Fig. 3 relaxation to the local charge neutral state can take place for low bias, since the value of $l_{c}$ is high enough. Similar behaviors are observed for other values of $l>1$ and $\alpha>1$.

The current-voltage characteristics for $l=50$ and several values of $\alpha$ are plotted in Fig. 4. The $I-V$ characteristics are 


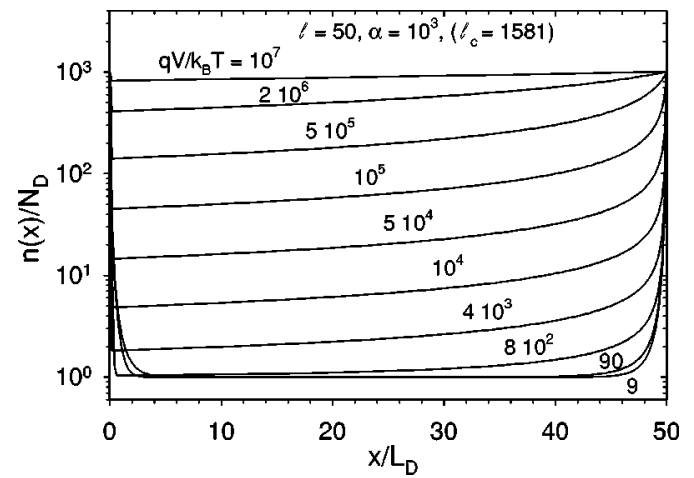

FIG. 3. Free carrier density profiles normalized to the donor density for $l$ $=50$ and $\alpha=10^{3}\left(l_{c}=1581\right)$, for several values of the applied bias.

linear at the lowest bias, superlinear at intermediate bias and again linear at the highest bias. This behavior is similar to that reported in Fig. 2 for the curves with $\alpha=10^{3}$ and $10^{4}$, since both cases correspond to situations where $l_{c}>1$.

In the present case $(l>1)$ one can reach an asymptotic limit for $l \gg 1$ and $\alpha \gg 1$ in which the following analytical asymptotic expression for the $I-V$ curve can be derived (see Appendix B):

$$
\frac{\bar{I}}{I_{\mathrm{th}}} \sim\left\{\begin{array}{l}
\frac{1}{l} \frac{q V}{k_{B} T}, \quad 0 \leqslant \frac{q V}{k_{B} T} \lesssim l^{2} \\
\frac{9}{8} \frac{1}{l^{3}}\left(\frac{q V}{k_{B} T}\right)^{2}, \quad l^{2} \lesssim \frac{q V}{k_{B} T} \lesssim \alpha l^{2}, \\
\frac{\alpha}{l} \frac{q V}{k_{B} T}, \quad \alpha l^{2} \lesssim \frac{q V}{k_{B} T}
\end{array}\right.
$$

where we have defined $I_{\mathrm{th}}=l k_{B} T /\left(q R_{\text {bulk }}\right)$. Equation (20) gives a good description of the $I-V$ curves for $l \gtrsim 10$ and $\alpha \gtrsim 100$, as can be seen in Fig. 4 where the symbols represent Eq. (20). ${ }^{12}$ Note that in the asymptotic limit, the low bias resistance is given by $R_{\text {low }}=R_{\text {bulk }}$, independent from any contact parameter since at low bias a state of local charge neutrality is achieved in the bulk of the sample (see Fig. 3). Furthermore, in the intermediate bias regime, the $I-V$ characteristics display a quadratic bias dependence, in

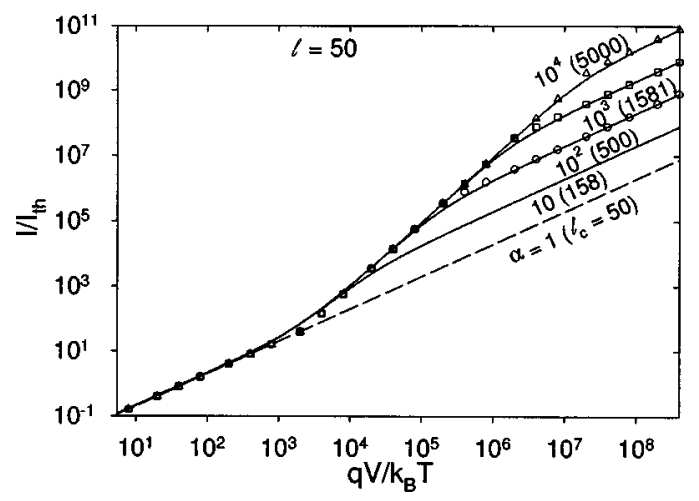

FIG. 4. Current-voltage characteristics for $l=50$ and several values of $\alpha$ (the corresponding value of $l_{c}$ is displayed for each curve). The dashed line corresponds to the homogeneous solution. The symbols correspond to the asymptotic expression of the $I-V$ characteristics given in Eq. (20). agreement with the well-known Mott and Gurney law for space charge transport in diffusive conductors. ${ }^{2}$ In this bias range, a strong injection of carriers from the contacts takes place, as illustrated in Fig. 3. Finally, at the highest voltages the resistance is given by $R_{\text {high }}=R_{c}$, since the density profile is almost homogeneous and equal to $n_{c}$ (see Fig. 3).

\section{CURRENT NOISE PROPERTIES}

We characterize the low frequency current fluctuation properties by means of the low frequency current spectral density defined as

$$
S_{I}(0)=2 \int_{-\infty}^{+\infty}\langle\delta I(0) \delta I(t)\rangle d t .
$$

For the model presented in Sec. III B, $S_{I}(0)$ can be given a closed analytical expression that takes into account in an exact way the effects of the diffusion current on the nonequilibrium current fluctuations and hence is applicable to the whole range of system parameters. Below we discuss separately the cases corresponding to homogeneous and inhomogeneous stationary density profiles, since they involve rather different mathematics.

\section{A. Homogeneous stationary profiles}

When the stationary profiles are homogeneous, Eq. (16) consists of a second order differential equation with constant coefficients. The solution of this equation, as well as an analytical expression for $S_{I}(0)$, has been derived recently: ${ }^{7}$

$$
\begin{aligned}
S_{I}(0)= & \frac{4 k_{B} T}{R_{\text {bulk }}}+K \frac{\left(\lambda_{2}^{2}-\lambda_{1}^{2}\right)}{2 L^{2} \lambda_{1}^{2} \lambda_{2}^{2}} \frac{\left(e^{\lambda_{1} L}-1\right)\left(e^{\lambda_{2} L}-1\right)}{\left(e^{\lambda_{2} L}-e^{\lambda_{1} L}\right)^{2}} \\
& \times\left[\lambda_{2}\left(e^{\lambda_{2} L}+1\right)\left(e^{\lambda_{1} L}-1\right)\right. \\
& \left.-\lambda_{1}\left(e^{\lambda_{1} L}+1\right)\left(e^{\lambda_{2} L}-1\right)\right],
\end{aligned}
$$

where $K=4 q A k_{B} T \mu \bar{n}$, and

$$
\lambda_{1,2}=-\frac{1}{2 L_{E}}\left(1 \pm \sqrt{1+4 \frac{L_{E}^{2}}{L_{D}^{2}}}\right)
$$

with $L_{E}=k_{B} T L /(q V)$. In Eq. (23) the subscript 1 corresponds to the plus term and the subscript 2 to the minus term.

For $l<1$, Eq. (22) takes the form ${ }^{7}$

$$
S_{I}(0)=2 q \bar{I} \operatorname{coth}\left(q V / 2 k_{B} T\right)
$$

Equation (24) predicts a standard crossover between Nyquist noise [Eq. (1)] and shot noise [Eq. (4)] for $q V / k_{B} T \sim 3$. The physical reason for the appearance of shot noise is the absence of long range Coulomb correlations due to the fact that the sample length $L$ is smaller than the Debye screening length $L_{D} \cdot{ }^{7}$

For $l>1$ the noise properties change considerably. Accordingly, in the limit $l \gg 1$ Eq. (22) is well approximated by ${ }^{7}$ 


$$
S_{I}(0)=S_{I}^{\mathrm{th}}\left\{\begin{array}{l}
\frac{4}{l} \text { for } 0 \leqslant \frac{\bar{I}}{I_{\mathrm{th}}} \lesssim l^{1 / 3} \\
\frac{2}{l^{2}}\left(\frac{\bar{I}}{I_{\mathrm{th}}}\right)^{3} \quad \text { for } l^{1 / 3} \lesssim \frac{\bar{I}}{I_{\mathrm{th}}} \leqq l, \\
2\left(\frac{\bar{I}}{I_{\mathrm{th}}}\right) \quad \text { for } l \leqq \frac{\bar{I}}{I_{\mathrm{th}}}
\end{array}\right.
$$

where $S_{I}^{\text {th }}=q I_{\text {th }}$. The above expression gives: Nyquist noise at low bias $\left(0 \leqslant q V / k_{B} T \leqq l^{4 / 3}\right)$, a cubic dependence on current of noise at intermediate bias $\left(l^{4 / 3} \lesssim q V / k_{B} T \lesssim l^{2}\right)$, and shot noise at the highest bias $\left(q V / k_{B} T \geqslant l^{2}\right)$. The physical reason for the appearance of shot noise in this case is the vanishing of long range Coulomb correlations because of a drift transit time shorter than both the diffusion transit time and the dielectric relaxation time. ${ }^{7}$

\section{B. Inhomogeneous stationary profiles}

When the stationary profile is inhomogeneous, Eq. (16) consists of a second-order stochastic differential equation with nonconstant coefficients. To obtain an analytical solution of this equation, we use a method developed in Refs. 13-15. The method is based on the fact that

$$
\rho(x)=d \bar{E}(x) / d x
$$

constitutes a particular solution of Eq. (16). On this basis one can find an analytical expression for the electric field fluctuations $\delta E_{x}(t)$ that solves Eq. (16) and satisfies the boundary condition in Eq. (17). ${ }^{15}$ From the expression of the electric field fluctuations we evaluate the voltage fluctuations, $\delta V(t)=\int_{0}^{L} \delta E_{x}(t) d x$. Following a procedure similar to that outlined in Ref. 15, the voltage fluctuations can be expressed as

$$
\delta V(t)=\int_{0}^{L} \nabla Z(x) \delta I_{x}(t) d x,
$$

with

$$
\begin{aligned}
\nabla Z(x)= & \frac{\rho(x)}{\epsilon A D}\left[\int_{x}^{L} \frac{E(\xi)-E_{\Delta}}{\rho^{2}(\xi)} e^{-q(\phi(x)-\phi(\xi)) / k_{B} T} d \xi\right. \\
& \left.+\frac{\left(E_{L}-E_{\Delta}\right)}{\rho_{L} \rho_{L}^{\prime}} e^{-q(V+\phi(x)) / k_{B} T}\right]
\end{aligned}
$$

and

$$
E_{\Delta}=\frac{\int_{0}^{L} \frac{E(x)}{\rho^{2}(x)} e^{q \phi(x) / k_{B} T} d x+\frac{E_{L}}{\rho_{L} \rho_{L}^{\prime}} e^{-q V / k_{B} T}-\frac{E_{0}}{\rho_{0} \rho_{0}^{\prime}}}{\int_{0}^{L} \frac{1}{\rho^{2}(x)} e^{q \phi(x) / k_{B} T} d x+\frac{1}{\rho_{L} \rho_{L}^{\prime}} e^{-q V / k_{B} T}-\frac{1}{\rho_{0} \rho_{0}^{\prime}}} .
$$

Here, $\phi(x)$ is the electric potential. The function $\nabla Z(x)$ is referred to as the impedance field, ${ }^{4,16,17}$ since it satisfies

$$
Z(0)=\int_{0}^{L} \nabla Z(x) d x=\left(\frac{d \bar{I}}{d V}\right)^{-1} .
$$

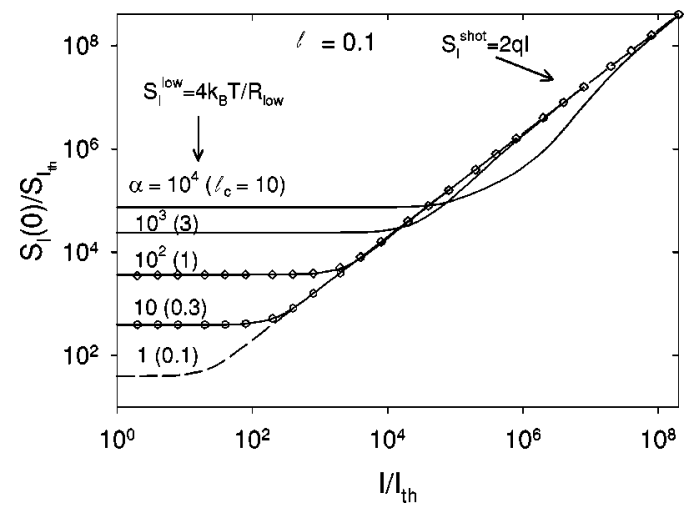

FIG. 5. Low frequency current spectral density normalized to $S_{I \text { th }}=q I_{\text {th }}$ as a function of the current normalized to $I_{\text {th }}$ for $l=0.1$ and several values of $\alpha$ (the corresponding value of $l_{c}$ is displayed for each curve). The dashed line corresponds to the homogeneous solution. The symbols correspond to the coth-like expression in Eq. (24).

From Eq. (27), the low frequency spectral density of the voltage fluctuations is given by

$$
S_{V}(0)=4 A q^{2} D \int_{0}^{L}[\nabla Z(x)]^{2} \bar{n}(x) d x,
$$

where use is made of Eqs. (12) and (13). The low frequency spectral density of current fluctuations is then evaluated from $^{10}$

$$
S_{I}(0)=\frac{S_{V}(0)}{Z(0)^{2}},
$$

Equations (28)-(32) constitute an exact solution of the noise model presented in Sec. III for the general case of an inhomogeneous stationary profile. They represent the main result of this article. Note that the solution incorporates the effects of the diffusion current without any approximation, thus allowing us to investigate the transition from homogeneous to highly inhomogeneous stationary profiles.

In order to evaluate Eqs. (28)-(32) we will use the steady-state profiles calculated in Sec. IV B. As for the case of transport we will consider the values $l=0.1$ and $l=50$, as representative examples of the behavior observed for $l<1$ and $l>1$.

Figure 5 reports the low frequency spectral density of current fluctuations $S_{I}(0)$ as a function of the current as obtained from Eqs. (28)-(32) for $l=0.1$ and several values of $\alpha$. For comparison the results for the homogeneous case, as calculated from Eq. (22), are also displayed (dashed line). We note that for the lowest degree of inhomogeneity ( $\alpha$ $\leqslant 10^{2}$ ) the nonequilibrium current noise results can be well approximated by Eq. (24) (symbols in Fig. 5). The reason is that for these values of $\alpha$ the profiles are quasihomogeneous with free carrier density $n_{c}$ and with Debye screening length satisfying $l_{c}<1$, which are the conditions for the validity of Eq. (24). Similar behaviors are observed for other values of $l<1$ and $\alpha>1$ for which $l_{c}<1$.

By contrast, for a high degree of inhomogeneity ( $\alpha$ $=10^{3}$ and $10^{4}$ ) the results deviate from Eq. (24), and regions where the current noise is suppressed below the shot noise limit are observed at intermediate biases values. The bias 


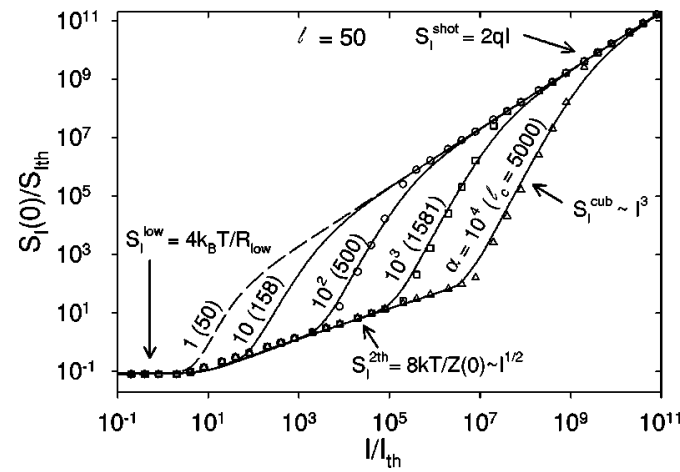

FIG. 6. Low frequency current spectral density normalized to $S_{I \text { th }}=q I_{\text {th }}$ as a function of the current normalized to $I_{\text {th }}$ for $l=50$ and several values of $\alpha$ (the corresponding value of $l_{c}$ is displayed for each curve). The dashed line corresponds to the homogeneous solution. The symbols correspond to the approximate expression in Eq. (33).

values where shot noise suppression takes place are found to correspond to those applied biases for which significant net carrier injection from the contacts takes place [see Fig. 1(b)]. The physical origin of the shot noise suppression should be traced back to the correlations induced by the long range Coulomb interaction which are active in this range of bias. Similar behaviors are observed for other values of $l<1$ and $\alpha>1$ for which $l_{c}>1$.

Figure 6 reports the low frequency spectral density of current fluctuations $S_{I}(0)$ as a function of the current as obtained from Eqs. (28)-(32) when $l=50$ and for several values of $\alpha$. For comparison, the results for the homogeneous case, as calculated from Eq. (22), are also displayed (dashed line). As can be seen, a qualitative new behavior at intermediate current values is identified with respect to the homogeneous behavior for all values of $\alpha$. The new behavior is found to tend to a square root dependence on the current and to cover broader current intervals as $\alpha$ is increased. This fact can be quantitatively evaluated by means of the following explicit expression valid for $l \gtrsim 10$ and $\alpha \gtrsim 100$ :

$$
\frac{S_{I}(0)}{S_{I}^{\text {th }}} \sim\left\{\begin{array}{l}
\frac{4}{l}, \quad 0 \lesssim \frac{\bar{I}}{I_{\mathrm{th}}} \lesssim \frac{l}{18} \\
\frac{12 \sqrt{2}}{l^{3 / 2}}\left(\frac{\bar{I}}{I_{\mathrm{th}}}\right)^{1 / 2}, \quad \frac{l}{18} \lesssim \frac{\bar{I}}{I_{\mathrm{th}}} \lesssim\left(\frac{9}{2} \alpha^{8} l\right)^{1 / 5} \\
\frac{8}{\alpha^{4} l^{2}}\left(\frac{\bar{I}}{I_{\mathrm{th}}}\right)^{3}, \quad\left(\frac{9}{2} \alpha^{8} l\right)^{1 / 5} \lesssim \frac{\bar{I}}{I_{\mathrm{th}}} \lesssim \frac{\alpha^{2} l}{2} \\
2\left(\frac{\bar{I}}{I_{\mathrm{th}}}\right), \quad \frac{\alpha^{2} l}{2} \lesssim \frac{\bar{I}}{I_{\mathrm{th}}}
\end{array}\right.
$$

(see Fig. 6 where the symbols correspond to the approximate expression). Otherwise, for $1<l<10$ and $1<\alpha<100$ the exact result should be used.

The dependence of the noise power as the square root of the current corresponds to the well known double thermal noise regime found in space charge limited devices ${ }^{4,5}$ [see Eq. (2)]. We note that the double thermal noise behavior is restricted to current values in the range $l / 18 \leq \bar{I} / I_{\text {th }}$ $\lesssim\left(4.5 \alpha^{8} l\right)^{1 / 5}$. For higher currents the noise properties devi-

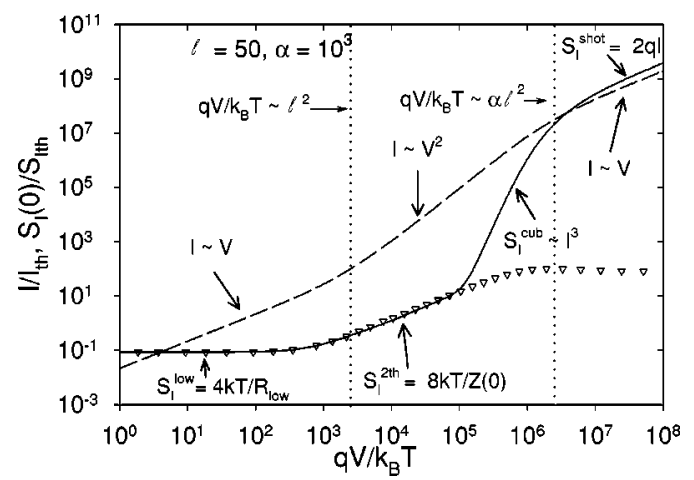

FIG. 7. Electric current (dashed line) and low frequency current spectral density (solid line) as a function of applied bias for $l=50$ and $\alpha=10^{3}$. The different behaviors are schematically indicated. The current is normalized to $I_{\text {th }}$ and the spectral density to $S_{I}^{\text {th }}=q I_{\text {th }}$. For comparison the results obtained from Eq. (3) are also displayed as triangles.

ate from the double thermal noise behavior and start resembling those corresponding to homogeneous conditions (cubic current dependence followed by shot noise). This fact is illustrated in Fig. 7 where we compare the exact results of the present article (continuous line) with those of existing theories for double thermal noise represented by Eq. (3) (triangles) for $l=50$ and $\alpha=10^{3}$. As can be seen by neglecting the diffusion current, existing theories can only be applied up to bias values below the onset of the cubic region. At applied bias above this onset, the diffusion current plays a relevant role and the exact theory presented here must be used. It is worth remarking that for devices operating under strong current injection conditions ( $\alpha \gtrsim 10^{4}$ ) the quasi-homogenous behavior predicted at the highest bias can be hardly observed in practice because of hot-carrier effects. ${ }^{18}$ However, in the low current injection regime $\left(1<\alpha \lesssim 10^{4}\right)$ this behavior should be experimentally accessible as discussed in the following suggested example.

\section{Example}

As an example to illustrate the experimental accessibility of the theoretical predictions described above we consider the following particular case. As semiconductor material we consider high resistive $p$-type $\mathrm{CdTe}$ at room temperature with a free carrier density (holes) $p=10^{8} \mathrm{~cm}^{-3}$. These low values of the free carrier density can be obtained by means of compensation. ${ }^{19}$ The hole mobility is taken to be $\mu \sim 40$ $\mathrm{cm}^{2} / \mathrm{V} \mathrm{s}$, the effective hole mass $m^{*}=0.8 m_{0}$, where $m_{0}$ is the electron mass, and the dielectric constant $\epsilon=10.3 \epsilon_{0}$, with $\epsilon_{0}$ being the vacuum permittivity. The sample length is assumed to be $L=4 \mathrm{~mm}$ and the cross sectional area $A$ $=40 \mathrm{~mm}^{2}$. As metal for the contacts we consider gold, which has been reported to form almost ideal metalsemiconductor junctions on CdTe. ${ }^{20}$ The value for the Au/ CdTe barrier height for holes, deduced from that for electrons, is $\phi_{b p}=0.55 \mathrm{~V}$, where use has been made of the relationship $\phi_{b p}=E_{g}-\phi_{b n}$ with $E_{g}=1.48 \mathrm{eV}$ and $\phi_{b n}$ $=0.93 \mathrm{~V}^{20}$ According to the value of $\phi_{b p}$ the contact density for holes is $p_{c}=10^{10} \mathrm{~cm}^{-3}$, where we used that $p_{c}$ $=N_{V} \exp \left[-q \phi_{b p} /\left(k_{B} T\right)\right]$, with $N_{V}=1.8 \times 10^{19} \mathrm{~cm}^{-3}$ being the CdTe effective density of the states in the valence band at 


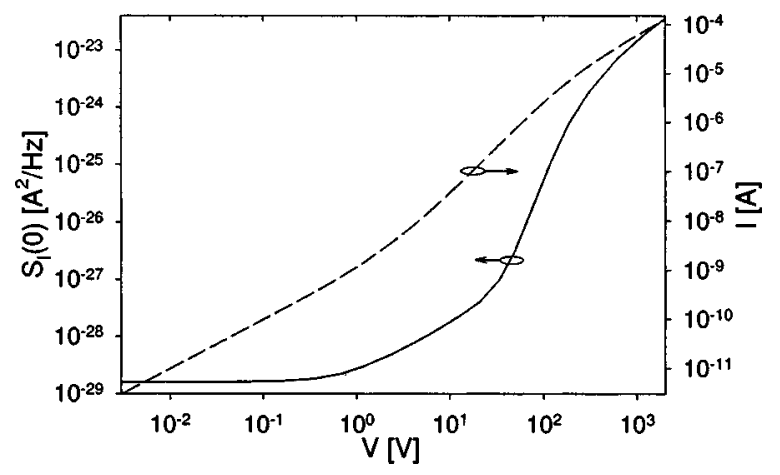

FIG. 8. Electric current (dashed line) and low frequency current spectral density (solid line) as a function of applied bias for a highly resistive $p$-type CdTe metal-semiconductor-metal structure. Parameters: free carrier density $10^{8} \mathrm{~cm}^{-3}$, sample length $4 \mathrm{~mm}$, cross sectional area $40 \mathrm{~mm}^{2}$, temperature $300 \mathrm{~K}$, and contact density $10^{10} \mathrm{~cm}^{-3}$.

room temperature. The metal contact described above forms an ohmic injecting contact in the terms described in the present article (see also the end of Appendix A).

For the set of parameters considered above, we have $L_{D}=0.4 \mathrm{~mm}$ and $L_{D c}=0.04 \mathrm{~mm}$, from where $l=L / L_{D}$ $=10, \alpha=n_{c} / N_{D}=100$ and $l_{c}=L / L_{D c}=100$. Figure 8 displays the calculated current $I$ (right axis) and low frequency current spectral density (left axis) as a function of the applied bias. According to the calculations, the predicted $I-V$ characteristic is linear up to bias voltages around $0.6 \mathrm{~V}$. For higher bias it tends to be quadratic, up to $V \sim 300 \mathrm{~V}$, from where it returns to linearity. Furthermore, the calculated current spectral density $S_{I}(0)$ displays the Nyquist thermal value up to voltages around $0.3 \mathrm{~V}$. Then, it increases with voltage according to the double thermal noise behavior up to around $20 \mathrm{~V}$. At further increasing voltages, $S_{I}(0)$ increases sharply with voltage according to the cubic dependence with applied current up to $V \sim 1 \mathrm{kV}$, where shot noise appears. It is worth noting that the calculated values of the low frequency current spectral density are well inside the range of experimental accessibility (state-of-the-art correlation spectrum analyzers ${ }^{21}$ are able to reach noise levels as low as $10^{-29} \mathrm{~A}^{2} / \mathrm{Hz}$ ). Moreover, the electric fields reach maximum values up to $E_{a v}=V / L=5 \mathrm{kV} / \mathrm{cm}$, which are still below that for the onset of hot electron effects in $p$-type CdTe. We conclude that such an example shows that the theoretical predictions presented in this article are accessible to an experimental confirmation.

\section{CONCLUSIONS}

We have carried out an analytical theory of transport and current fluctuation properties in metal-semiconductor-metal structures made of highly resistive semiconductors. The theory includes the effects of the diffusion current in an exact way, thus allowing us to study the whole range of physical conditions concerning the strength of the applied bias and the level of carrier injection from the contacts. It is shown that in the low carrier injection limit for which $l_{c}=L / L_{D c}<1$, where $L$ is the sample length and $L_{D c}$ the Debye screening length associated with the free carrier density at the metalsemiconductor interface $n_{c}$, the structure behaves like a lin- ear resistor with low frequency noise properties given by $S_{I}(0)=2 q \bar{I} \operatorname{coth}\left[q V /\left(2 k_{B} T\right)\right]$. In the intermediate carrier injection regime, roughly determined by $1<l_{c} \lesssim 100$, the structure displays linear-superlinear-linear current-voltage characteristics. In this regime, the current spectral density displays a crossover from Nyquist noise to shot noise mediated by a region depending first as the square root of the current (double thermal noise) and then as the third power of the current. Finally, under strong carrier injection conditions $l_{c} \gg 1$ the standard theory of space charge limited diodes is recovered. In this limit the current-voltage characteristics are first linear and then quadratic. Accordingly, the current spectral density displays Nyquist thermal noise at low bias followed by double thermal noise at higher applied bias. We suggest that high resistive $p$-type CdTe is one of the best suited materials to provide an experimental test of the theoretical predictions in the small and moderated injection regimes.

\section{ACKNOWLEDGMENTS}

Partial support from the MCyT-Spain through the Ramon y Cajal program and Projects No. BFM2000-0624 and No. BFM2001-2159 and from the Italy-Spain Joint Action of the MIUR-Italy (Ref. IT109) and MCyT-Spain (Ref. HI20000138) is gratefully acknowledged. Professor T. Gonzalez of Salamanca University and Professor M. Sampietro of Politecnico di Milano are acknowledged for stimulating discussions carried out on the subject.

\section{APPENDIX A: METAL-SEMICONDUCTOR MODEL CONTACT}

In this appendix we justify the boundary conditions used along the article for both the transport, Eq. (7), and the noise properties, Eq. (15). Following Refs. 1, 22, and 23, the general boundary conditions for the transport through a metalsemiconductor contact read

$$
\bar{n}(0)=\frac{\bar{I}+I_{s}^{0}}{q A v_{r}^{0}}, \quad \bar{n}(L)=\frac{-\bar{I}+I_{s}^{L}}{q A v_{r}^{L}},
$$

with $v_{r}^{0}$ and $I_{s}^{0}$ (respectively, $v_{r}^{L}$ and $I_{s}^{L}$ ) being the recombination velocity and saturation current, respectively, of the contact located at $x=0$ (respectively $x=L)$. The currents $I_{s}^{L}$ and $I_{s}^{0}$ are given by $I_{s}^{0}=q A v_{r}^{0} n_{0}^{\mathrm{eq}}$ and $I_{s}^{L}=q A v_{r}^{L} n_{L}^{\mathrm{eq}}$, where

$$
n_{0}^{\mathrm{eq}}=N_{C} e^{-q \phi_{b n}^{0} / k_{B} T}, \quad n_{L}^{\mathrm{eq}}=N_{C} e^{-q \phi_{b n}^{L} / k_{B} T},
$$

Here, $N_{C}$ is the effective density of states in the conduction band and $\phi_{b n}^{0}\left(\phi_{b n}^{L}\right)$ is the barrier height at contact $x=0$ $(x=L)$. For the case of ideal metal-semiconductor junctions, the barrier heights are given by $\phi_{b n}^{0}=\phi_{m}^{0}-\chi$ and $\phi_{b n}^{L}=\phi_{m}^{L}-\chi$, with $\chi$ being the semiconductor affinity and $\phi_{m}^{0}\left(\phi_{m}^{L}\right)$ the work function of the metal located at $x=0(x$ $=L)$. For highly resistive materials the diffusion approximation $^{1}$ can be applied. In this approximation it is assumed that the current takes values much smaller than the thermionic current, i.e., $\bar{I} \ll I_{s}$. This condition implies ${ }^{14}$ $\bar{I} / I_{\mathrm{th}} \ll \bar{I}_{s} / I_{\mathrm{th}}=\alpha / \beta$, where 


$$
\beta=\mu \sqrt{\frac{2 \pi N_{D} m^{*}}{\epsilon}}, \quad \alpha=\frac{n^{\mathrm{eq}}}{N_{D}} .
$$

In this limit, one can approximate Eq. (A1) by

$$
\bar{n}(0) \sim \frac{I_{s}^{0}}{q A v_{r}^{0}}=n_{0}^{\mathrm{eq}}, \quad \bar{n}(L) \sim \frac{I_{s}^{L}}{q A v_{r}^{L}}=n_{L}^{\mathrm{eq}},
$$

which for the case of a symmetric structure corresponds to the boundary conditions in Eq. (7). This result also implies that the resistance of the metal-semiconductor interface is negligibly small in comparison to the bulk resistance. As a result, the fluctuations generated at the metal-semiconductor interface can be completely neglected. This is equivalent to approximate $^{14}$

$$
\delta n_{0}(t) \sim 0, \quad \delta n_{L}(t) \sim 0,
$$

in agreement with the boundary conditions in Eq. (15).

For the concrete example considered in Sec. V C, one has $\beta=9 \times 10^{-6} \ll 1$, which allows us to apply the diffusive approximation up to currents satisfying the condition $I_{s} / I_{\text {th }}$ $=\alpha / \beta=1.1 \times 10^{7}$, well above the current values required to observe the different behaviors here identified.

\section{APPENDIX B: ASYMPTOTIC TRANSPORT THEORY}

In this appendix we derive the asymptotic expression for the $I-V$ characteristics used in Eq. (20) of Sec. IV B. To this purpose, we start by considering the limit when $l \gg 1$. In this limit, one can neglect the diffusive contribution in Eq. (9), thus obtaining a first order differential equation for the electric field of the form

$$
-\bar{E}(x)\left[\frac{d \bar{E}(x)}{d x}-1\right]=\bar{I},
$$

subject to the boundary condition $E(L)=\bar{I} / \alpha$. In Eq. (B1), and along this appendix, we use dimensionless variables to simplify the expressions. Accordingly, we take $\bar{E} \rightarrow \bar{E} / E_{\text {th }}$, $\bar{I} \rightarrow \bar{I} / I_{\text {th }}, \quad x \rightarrow x / L_{D}, L \rightarrow L / L_{D}, \quad \bar{U}(x) \rightarrow \bar{U}(x) / k_{B} T$, where $E_{\mathrm{th}}=\left(k_{B} T N_{D} / \epsilon\right)^{1 / 2}$. Equation (B1) can be easily integrated between $x=0$ and $x=L$ to give the following relation

$$
\bar{E}_{L}+\bar{I} \ln \left(1-\frac{\bar{E}_{L}}{\bar{I}}\right)-\bar{E}_{0}+\bar{I} \ln \left(1-\frac{\bar{E}_{0}}{\bar{I}}\right)=L,
$$

where $\bar{E}_{L}=\bar{E}(L)$ and $\bar{E}_{0}=\bar{E}(0)$. On the other hand, by using again Eq. (B1), one can derive an explicit expression for the potential energy difference $\bar{U}(x)-\bar{U}_{L}=\int_{L}^{x} \bar{E}(x) d x$, which once evaluated at $x=0$ gives for the applied bias, $V$ $=U_{L}-U_{0}$,

$$
\begin{aligned}
\bar{V}= & \frac{\bar{E}_{L}^{2}}{2}+\overline{I E}_{L}+\bar{I}^{2} \ln \left(1-\frac{\bar{E}_{L}}{\bar{I}}\right) \\
& -\frac{\bar{E}_{0}^{2}}{2}-\overline{I E}_{0}-\bar{I}^{2} \ln \left(1-\frac{\bar{E}_{0}}{\bar{I}}\right) .
\end{aligned}
$$

To end up with an explicit expression for the $I-V$ characteristics, we impose the boundary condition $\bar{E}_{L}=\bar{I} / \alpha$ in Eqs. (B3) and (B2), thus giving the $I-V$ characteristics in parametric form:

$$
\bar{V}=\frac{\left[\frac{1}{2 \alpha^{2}}+\frac{1}{\alpha}+\ln \left(1-\frac{1}{\alpha}\right)-\frac{u_{0}^{2}}{2}-u_{0}-\ln \left(1-u_{0}\right)\right]}{L^{2}\left[\frac{1}{\alpha}+\ln \left(1-\frac{1}{\alpha}\right)-u_{0}-\ln \left(1-u_{0}\right)\right]^{2}},
$$

$$
\bar{I}=\frac{L}{\left[\frac{1}{\alpha}+\ln \left(1-\frac{1}{\alpha}\right)-u_{0}-\ln \left(1-u_{0}\right)\right]},
$$

where the parameter $u_{0}=\bar{E}_{0} / \bar{I}$ satisfies the condition $1 / \alpha$ $<u_{0}<1$. By further expanding the previous expression for $\alpha \gg 1$ one then arrives at Eq. (20).

${ }^{1}$ S. M. Sze, Physics of Semiconductor Devices, 2nd ed. (Wiley, New York, 1981).

${ }^{2}$ M. A. Lampert and P. Mark, Current Injection in Solids (Academic, New York, 1972).

${ }^{3}$ H. Nyquist, Phys. Rev. B 32, 110 (1928).

${ }^{4}$ K. M. van Vliet, A. Friedmann, R. J. J. Ziljstra, A. Gisoft, and A. van der Ziel, J. Appl. Phys. 46, 1804 (1975); 46, 1814 (1975).

${ }^{5}$ N. A. Nicolet, H. R. Bliger, and R. J. J. Ziljstra, Phys. Status Solidi B 70, 9 (1975); 70, 415 (1975).

${ }^{6}$ A. van der Ziel, Semicond. Semimet. 14, 195 (1979).

${ }^{7}$ G. Gomila and L. Reggiani, Phys. Rev. B 62, 8068 (2000).

${ }^{8}$ J. P. Nougier, D. Gasquet, J. C. Vaissiere, and H. R. Bilger, Proceedings of the 5th International Conference on Noise in Physical Systems, edited by D. Wolf (Springer-Verlag, Berlin, 1978), p. 110.

${ }^{9}$ A. van der Ziel, Noise in Solid State Devices and Circuits (Wiley, New York, 1985).

${ }^{10}$ C. M. van Vliet, IEEE Trans. Electron Devices 41, 1902 (1994).

${ }^{11}$ F. Bonani and G. Ghione, Noise in Semiconductors Devices, Modeling and Simulation (Springer-Verlag, Berlin, 2001).

${ }^{12}$ For values of $l \gtrsim 10$ and $\alpha<100$ one can use the parametric expression for the $I-V$ derived in Appendix B, Eqs. (B4) and (B5).

${ }^{13}$ O. M. Bulashenko, G. Gomila, J. M. Rubí, and V. A. Kochelap, Appl. Phys. Lett. 70, 3248 (1997); J. Appl. Phys. 83, 2610 (1998).

${ }^{14}$ G. Gomila, O. M. Bulashenko, and J. M. Rubí, J. Appl. Phys. 83, 2619 (1998).

${ }^{15}$ G. Gomila and O. M. Bulashenko, J. Appl. Phys. 86, 1004 (1999).

${ }^{16}$ W. Shockley, J. A. Copeland, and R. P. James, in Quantum Theory of Atoms Molecules and the Solid State, edited by P. O. Lowdin (Academic, New York 1966), p. 537.

${ }^{17}$ P. Shiktorov, E. Starikov, V. Gruzhinskis, T. Gonzalez, J. Mateos, D. Pardo, L. Reggiani, L. Varani, and J. C. Vaissiere, Riv. Nuovo Cimento 24, 1 (2001).

${ }^{18}$ L. Reggiani, in Hot-Electron Transport in Semiconductor (SpringerVerlag, Berlin, 1985).

${ }^{19}$ A. J. Strauss, Rev. Phys. Appl. 12, 167 (1997).

${ }^{20}$ I. M. Dharmadasa, J. M. Thorton, and R. H. Williams, Appl. Phys. Lett. 54, 137 (1989).

${ }^{21}$ M. Sampietro, L. Fasoli, and G. Ferrari, Rev. Sci. Instrum. 70, 2520 (1999).

${ }^{22}$ G. Gomila, A. Pérez-Madrid, and J. M. Rubí, Physica A 233, 208 (1996).

${ }^{23}$ G. Gomila, J. M. Rubí, I. R. Cantalapiedra, and L. L. Bonilla, Phys. Rev. E 56, 1490 (1997). 